This is an electronic reprint of the original article. This reprint may differ from the original in pagination and typographic detail.

Author(s): Virtanen, Pauli; Heikkilä, Tero

Title: $\quad$ Spin Pumping and Torque Statistics in the Quantum Noise Limit

Year: $\quad 2017$

Version:

Please cite the original version:

Virtanen, P., \& Heikkilä, T. (2017). Spin Pumping and Torque Statistics in the Quantum Noise Limit. Physical Review Letters, 118(23), Article 237701. https://doi.org/10.1103/PhysRevLett.118.237701

All material supplied via JYX is protected by copyright and other intellectual property rights, and duplication or sale of all or part of any of the repository collections is not permitted, except that material may be duplicated by you for your research use or educational purposes in electronic or print form. You must obtain permission for any other use. Electronic or print copies may not be offered, whether for sale or otherwise to anyone who is not an authorised user. 


\title{
Spin Pumping and Torque Statistics in the Quantum Noise Limit
}

\author{
P. Virtanen ${ }^{1,2}$ and T. T. Heikkilä ${ }^{2}$ \\ ${ }^{1}$ NEST, Istituto Nanoscienze-CNR and Scuola Normale Superiore, I-56127 Pisa, Italy \\ ${ }^{2}$ University of Jyvaskyla, Department of Physics and Nanoscience Center, P.O. Box 35, 40014 University of Jyväskylä, Finland
}

(Received 29 September 2016; revised manuscript received 21 March 2017; published 8 June 2017)

\begin{abstract}
We analyze the statistics of charge and energy currents and spin torque in a metallic nanomagnet coupled to a large magnetic metal via a tunnel contact. We derive a Keldysh action for the tunnel barrier, describing the stochastic currents in the presence of a magnetization precessing with the rate $\Omega$. In contrast to some earlier approaches, our result is valid for an arbitrary ratio of $\hbar \Omega / k_{B} T$. We illustrate the use of the action by deriving spintronic fluctuation relations, the quantum limit of pumped current noise, and consider the fluctuations in two specific cases: the situation with a stable precession of magnetization driven by spin transfer torque, and the torque-induced switching between the minima of a magnetic anisotropy. The quantum corrections are relevant when the precession rate exceeds the temperature $T$, i.e., for $\hbar \Omega \gtrsim k_{B} T$.
\end{abstract}

DOI: 10.1103/PhysRevLett.118.237701

Spin transfer torque, angular momentum contributed by electrons entering a magnet, can be used to control magnetization dynamics via electrical means, as demonstrated in many experiments [1-3]. Often the effect can be described by considering the ensemble average magnetization dynamics, or taking only thermal noise into account [4]. The spin transfer torque is in general also a stochastic process, but at bias voltages large enough to drive the magnetization, it is not necessarily Gaussian nor thermal [5], especially at cryogenic temperatures. The statistical distribution of electron transfer and the associated torque in magnetic tunnel junctions can be described by counting statistics [6], via a joint probability distribution $P_{t_{0}}(\delta n, \delta E, \delta s)$ of charge $\delta n$, energy $\delta E$, and spin $\delta s$ transferred into the magnet during time $t_{0}$. The distribution is conditional on the magnetization dynamics during time $t_{0}$, which necessitates consideration of backaction effects.

Here we construct a theory describing the probability distribution for electron transfer via a Keldysh action [Eq. (3)] describing a metallic magnet with magnetization $\boldsymbol{M}$, coupled to a fermionic reservoir (another ferromagnetic metal), illustrated in Fig. 1. In the presence of a bias voltage in the reservoir, this coupling may lead to a stochastic spin transfer torque affecting the magnetization dynamics. Unlike some of the earlier discussions of counting and spin torque statistics [7-10], we follow the approach of Ref. [11] to derive the relevant tunneling spin action $[12,13]$, and retain geometric phase factors in the derivation of the generating function. The phase factors become relevant in the quantum limit $\hbar \Omega>k_{B} T$, where the precession rate $\Omega$ is large compared to the temperature $T$. In addition, we show how this quantum limit can be accessed via the measurement of the current noise or the switching rate of magnetization.

In particular, we suggest two specific settings [Figs. 1(b), 1(c)], characterized by opposite regimes of the external field $\mathbf{H}_{\mathrm{ext}}$ and anisotropy field $\mathbf{H}_{\mathrm{an}}$. When $H_{\mathrm{ext}} \gg H_{\mathrm{an}}$, a suitably chosen voltage drives the magnet into a stationary precession with rate $\Omega$ around the direction of $\mathbf{H}_{\mathrm{ext}}$
[1,14-16]. This precession pumps charge [17] and heat into the reservoir, along with the direct charge and heat currents due to the applied voltage. The noise of these currents depends on the intrinsic noise of the pumped current and, at low frequencies, also on the fluctuations of the magnetization, driven by the spin torque noise. The opposite limit $H_{\text {an }} \gg H_{\text {ext }}$ is the one relevant for memory applications, as the spin transfer torque can be used to switch between the two stable magnetization directions $[18,19]$. Our approach allows finding the switching rate at any temperature and voltage, also for $k_{B} T \ll \hbar \Omega$.

Besides the average currents and noise, the Keldysh action allows us to calculate the full probability distribution $P_{t_{0}}(\delta n, \delta E, \delta s)$ of transmitted charge $\delta n$, energy $\delta E$, or change $\delta M_{z}=\mathcal{S} \gamma \delta s / \mathcal{V}$ of the $z$ component of magnetization (a)

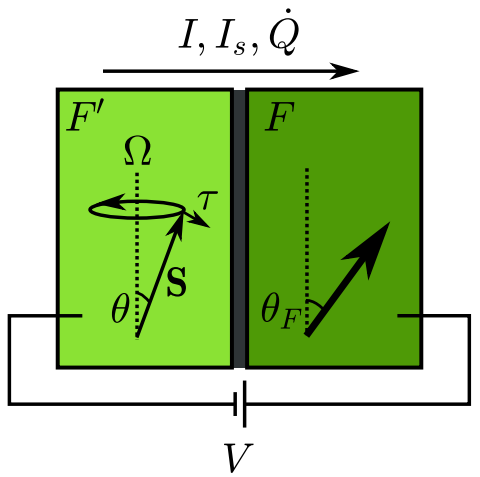

(b)

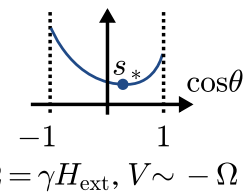

(c)

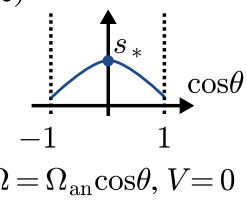

FIG. 1. (a) Tunnel junction between magnetic materials with free $\left(F^{\prime}\right)$ and fixed $(F)$ magnetizations, biased by voltage $V$. The total spin $\boldsymbol{S}=\mathcal{V} \boldsymbol{M} / \gamma$ in $F^{\prime}$ precesses at angular frequency $\Omega$ around the $z$ axis. As described by Eq. (3), the motion pumps charge, spin, and heat currents through the junction, and the backaction spin transfer torque $\boldsymbol{\tau}$ drives a change in the tilt angle $\theta$. (b)-(c) Schematic of the effective magnetic potential energy, in the presence of an external field $H_{\text {ext }}$ and large spin transfer torque, or, in the presence of a magnetic anisotropy $\Omega_{\mathrm{an}}=\gamma H_{\mathrm{an}}$. 
in a nanomagnet with volume $\mathcal{V}$ and spin $\mathcal{S} \gg 1$, within a long measurement time $t_{0}$. Here, $\gamma$ is the gyromagnetic ratio. The precise distribution depends on the exact driving conditions and the parameters of the setup. However, symmetries constrain the probability distribution, leading to a spintronic fluctuation relation (here and below, $k_{B}=\hbar=e=1$ )

$$
\begin{aligned}
& P_{t_{0}}(\delta n, \delta E, \delta s) \\
& \quad=e^{V \delta n / T_{F}} e^{\delta E\left(T_{F}^{-1}-T_{F^{\prime}}^{-1}\right)} e^{-\Omega \delta s / T_{F}} P_{t_{0}}^{\prime}(-\delta n,-\delta E, \delta s),
\end{aligned}
$$

where $P_{t_{0}}^{\prime}$ corresponds to the case with reversed magnetizations. Here, $V$ is the voltage across the junction, and $T_{F}$ and $T_{F^{\prime}}$ the electronic temperatures on the two sides. As in fluctuation relations presented earlier [7,20-27], this allows for a direct derivation of Onsager symmetries, thermodynamical constraints, and fluctuation-dissipation relations, valid for the coupled charge-spin-energy dynamics [28].

Generating function.-Consider a magnetic tunnel junction depicted in Fig. 1. The spin transfer torque due to tunnelling, and the corresponding counting statistics can be described by a Keldysh action obtained by integrating out conduction electrons in $F$ and $F^{\prime}$ [9,11]. We apply the approach of Ref. [11] to the characteristic function $Z(\chi, \xi)=\left\langle e^{i\left\{N_{F}\left(t_{0}\right) \chi+\left[H_{F}\left(t_{0}\right)-\mu\right] \xi\right\}} e^{-i\left\{N_{F}(0) \chi+\left[H_{F}(0)-\mu\right] \xi\right\}}\right\rangle$ describing the change in particle number $N_{F}$ and internal energy $H_{F}$ relative to chemical potential $\mu$ in the ferromagnetic lead $F$ [23,33]. In the long-time limit, $t_{0} \gg 1 / T, 1 / V$, this results to the action $S=S_{0}+S_{T}$, where $S_{0}=\mathcal{S} \int_{-\infty}^{\infty} d t\left[-2 \dot{\psi}^{q}-\sum_{ \pm}\left( \pm \dot{\phi}^{c l}+\dot{\phi}^{q}\right) \cos \left(\theta^{c l} \pm \theta^{q}\right)\right]$ is the Berry phase for total spin $\mathcal{S}=\mathcal{V}|\boldsymbol{M}| / \gamma$. Moreover, the tunneling action is

$S_{T}=i|W|^{2} \int_{-\infty}^{\infty} d t d t^{\prime} \frac{d \epsilon}{2 \pi} \operatorname{Tr} \check{P}(t) \check{G}_{F^{\prime}}\left(t-t^{\prime}\right) \check{P}\left(t^{\prime}\right)^{\dagger} \check{G}_{F}(\epsilon)$,

where $\check{P}(t)=e^{i(\epsilon-V) t} e^{i[\chi(t)+(\epsilon-\mu) \xi(t)] \check{Y}_{x} / 2} \check{R}(t)$ contains the bias voltage $V$, and the charge and energy counting fields $\chi(t)$ and $\xi(t)$. The rotation matrix $\check{R}(t)=$ $e^{-i \check{\phi}(t) \sigma_{z} / 2} e^{-i \check{\theta}(t) \sigma_{y} / 2} e^{-i \check{\psi}(t) \sigma_{z} / 2}$ describes the direction of the magnetization $S=(\cos \phi \sin \theta, \sin \phi \sin \theta, \cos \theta) \mathcal{S}$ in terms of Euler angles $\theta$ and $\phi$. Keldysh fields are in the basis [34] $\check{\phi}=\phi^{c l}+\phi^{q} \check{\gamma}_{x}$, where $\check{\gamma}_{x}$ is a Pauli matrix. The above result assumes the gauge is fixed [11] as $\psi^{q}=-\phi^{q} \cos \theta^{c l}, \dot{\psi}^{c l}=-\dot{\phi}^{c l} \cos \theta^{c l}$. We assume a spin and momentum independent tunneling matrix element $W$. The conduction electrons are described by Keldysh Green functions $\breve{G}$, with the exchange field of $F^{\prime}$ always parallel to $\hat{z}$ in the rotating frame; in momentum representation the retarded component reads $\breve{G}_{F^{\prime}}^{R}(\epsilon, \boldsymbol{k})=\left[\epsilon+i 0^{+}-\right.$ $\left.\xi_{k}+h_{F^{\prime}} \sigma_{z}\right]^{-1}$, where $\xi_{k}$ is the energy dispersion and $h_{F^{\prime}}$ an internal exchange field (see Ref. [28] for details).

Consider now the situation depicted in Fig. 1(a), where $S$ precesses around $\hat{z}$ due to an external magnetic field and/or magnetic anisotropy contributing potential energy $E_{M}$. The corresponding action is $S_{\text {ext }}=\int d t \sum_{ \pm} \pm E_{M}\left[S_{ \pm}\right]=$ $2 \int d t \boldsymbol{S}^{q} \cdot \hat{z} \Omega$, with $\Omega=\Omega_{\mathrm{ext}}+\Omega_{\mathrm{an}}[\cos \theta]^{c l}$. Separating out the fast motion $\phi^{c l}(t)=\Omega t+\tilde{\phi}^{c l}(t)$, the dynamics of $\theta, \tilde{\phi}$ are driven only by the spin transfer torque. We assume this dynamics is slow, and evaluate Eq. (2) under a time scale separation $\sim t_{0}^{-1},|W|^{2} / \mathcal{S} \ll T, \Omega$ :

$$
S_{T} \simeq-i \int_{-\infty}^{\infty} d t d \epsilon \sum_{\sigma \sigma^{\prime} \alpha= \pm} \Gamma_{\sigma \sigma^{\prime} \alpha}(\epsilon)\left(e^{i \alpha \eta_{\sigma \sigma^{\prime}}(\epsilon)}-1\right) .
$$

Here, $\eta_{\sigma \sigma^{\prime}}(\epsilon)=\chi(t)+\left(\epsilon-\mu+V+\Omega_{\sigma \sigma^{\prime}}\right) \xi(t)-2\left(\Omega_{\sigma \sigma^{\prime}} / \Omega\right) \phi^{q}(t)$ and $\Omega_{\sigma \sigma^{\prime}}=\left[\sigma \Omega-\sigma^{\prime} \Omega \cos \theta^{c l}(t)\right] / 2$. The transition rates per energy are

$$
\begin{gathered}
\Gamma_{\sigma \sigma^{\prime} \alpha}(\epsilon)=\bar{G}_{\sigma \sigma^{\prime}} \frac{1+\sigma \sigma^{\prime} \cos \theta^{c l}(t)}{2} \Lambda_{\alpha}\left(\epsilon, V+\Omega_{\sigma \sigma^{\prime}}\right), \\
\Lambda_{\alpha}(\epsilon, V)= \begin{cases}f_{F^{\prime}}(\epsilon)\left[1-f_{F}(\epsilon+V)\right], & \alpha=+, \\
f_{F}(\epsilon+V)\left[1-f_{F^{\prime}}(\epsilon)\right], & \alpha=-.\end{cases}
\end{gathered}
$$

Here, $f_{F / F^{\prime}}(\epsilon)=1 /\left[e^{(\epsilon-\mu) / T_{F / F^{\prime}}}+1\right]$ are Fermi distribution functions, and the time-averaged conductance is $\bar{G}_{\sigma \sigma^{\prime}}=G_{0}\left\{\left[\left(1+\sigma P_{F z}\right) / 2\right]\left[\left(1+\sigma^{\prime} P_{F^{\prime}}\right) / 2\right]\right\}$, where $G_{0}=$ $2 \pi|W|^{2}\left(\nu_{F \uparrow}+\nu_{F \downarrow}\right)\left(\nu_{F^{\prime} \uparrow}+\nu_{F^{\prime} \downarrow}\right)$, the polarizations are defined as $P=\left(\nu_{\uparrow}-\nu_{\downarrow}\right) /\left(\nu_{\uparrow}+\nu_{\downarrow}\right)$, and $P_{F z}=P_{F} \cos \theta_{F}$ is the polarization of the fixed magnet projected onto the precession axis. The densities of states $\nu_{\uparrow / \downarrow}$ of majority or minority spins are given at the Fermi level. The resulting $S_{T}$ is independent of $\tilde{\phi}^{c l}$; i.e., its dynamics decouples, which constrains $\theta^{q}=0$ [28].

The result describes Poissonian transport events, each associated with a backaction on $\theta$ due to the spin transfer torque, as described by the dependence on $\phi^{q}$. The rates are proportional to the averaged densities of states and squared spin overlaps $\left|\left\langle\sigma \mid \sigma^{\prime}\right\rangle\right|^{2}=\left[1+\sigma \sigma^{\prime} \cos \theta\right] / 2$ in the frame rotating with the magnetic precession. The transferred energy $V+\Omega_{\sigma \sigma^{\prime}}$ consists of the voltage bias and the difference $\pm \Omega / 2-( \pm \Omega \cos \theta) / 2$ of energy shifts on the right and left sides of the junction in the rotating frame $[2,35]$. The relation of this additional dependence on $\theta$ to geometric phases is discussed in Ref. [11]. It also separates Eq. (3) from the result of Ref. [7] for tunneling through a ferromagnetic insulator barrier, where such angular dependencies are not included. In particular, the above results imply assumptions on relaxation time scales: conduction electrons are assumed to follow adiabatically the slowly varying internal exchange field $\boldsymbol{h}_{F^{\prime}} \| \boldsymbol{S}$, and relaxation is assumed to be fast compared to, e.g., the injection rates $\propto|W|^{2} V$ in order to maintain the equilibrium form of the electron distribution.

Equation (3) is a main result of this work, as the knowledge of $S_{T}$ allows access to the statistics of charge, energy, and spin transfer in the generic case depicted in Fig. 1(a). Below, we describe some applications. First, we can identify the following spintronic fluctuation relation [28] 
$S_{T}\left(\chi, \xi, \phi^{q}\right)=S_{T}^{\prime}\left(-\chi+\frac{i V}{T_{F}},-\xi+\frac{i}{T_{F}}-\frac{i}{T_{F^{\prime}}}, \phi^{q}+\frac{i \Omega}{2 T_{F}}\right)$,

where the prime denotes inverting the magnetizations and the sign of the precession. Identifying the conjugate fields of $\chi, \xi$, and $\phi^{q}$ to the number of charges $\delta n$, change of energy $\delta E$ and transfer of spin angular momentum $\delta s$, this relation is equivalent with Eq. (1). This relation also implies the Onsager relation $d I / d \Omega=\left(\sin ^{2} \theta\right) d \tau / d V$ relating the pumped current to the torque $\tau \sin ^{2} \theta \equiv d S_{T} / d\left(2 \phi^{q}\right)$ acting on the angle $\theta$ [36]. This and further details of the fluctuation relation are discussed in Ref. [28].

The average dynamics follows the $\theta$ component of the Landau-Lifshitz-Slonczewski equation, [1] here obtained from stationarity of $S$ vs $\phi^{q}$,

$$
\dot{S} \dot{\theta}=-\sin (\theta) \tau(\theta), \quad \tau(\theta)=\Omega \alpha(\theta)+I_{s z},
$$

where the spin current $I_{s z}=\frac{1}{4} G_{0} P_{F z} V$ and damping $\alpha(\theta)=\frac{1}{8} G_{0}\left[1-P_{F_{z}} P_{F^{\prime}} \cos \theta\right]$ [37] have been discussed in Ref. [9]. The equation describes motion of $\cos \theta$ in an effective potential $-\int{ }^{\cos \theta} d\left(\cos \theta^{\prime}\right) \tau\left(\theta^{\prime}\right)$ defined by $\Omega(\theta)$ and the spin torque, illustrated in Fig. 1(b). In certain parameter ranges, a fixed point $\tau\left(\theta_{*}\right)=0$ appears-it can be either attractive or repulsive. This can correspond to a stable but fluctuating precession around the angle $\theta_{*}$ [Fig. 1(b)], induced by spin torque, or spin torque-induced switching between two energy minima [Fig. 1(c)].

Average current and noise.-For fast measurements, $t_{0} \ll 1 / \dot{\theta}$, we can assume $\theta$ remains fixed, and find the average charge $\bar{I}$ and heat $\overline{\dot{Q}_{F}}$ currents entering $F$ during time $t_{0}$,

$$
\begin{gathered}
\bar{I}=\frac{1}{2} G_{0}\left[1+P_{F^{\prime}} P_{F z} \cos \theta\right] V+\frac{1}{4} G_{0} P_{F z} \Omega \sin ^{2} \theta, \\
\overline{\dot{Q}_{F}}=\frac{1}{2} \bar{I} V+\frac{1}{2} \tau(\theta) \Omega \sin ^{2} \theta,
\end{gathered}
$$

where the pumped charge current [second term in Eq. (8)] is that found in Ref. [35]. The heat current is a sum of the Joule heat and the magnetic energy lost due to the spin torque, $\dot{E}_{M}=-\partial_{t}\left[\Omega_{\mathrm{ext}} S_{z}+\frac{1}{2} \Omega_{\mathrm{an}} S_{z}^{2}\right]$, dissipated equally in $F$ and $F^{\prime}$. In contrast to the average values, the energy shifts $\Omega_{\sigma \sigma^{\prime}}$ remain in the zero-frequency noise $S_{I}, S_{\dot{Q}_{F}}$ of the charge and heat current,

$$
\begin{gathered}
S_{I}=\sum_{\sigma \sigma^{\prime}} \bar{G}_{\sigma \sigma^{\prime}} \frac{1+\sigma \sigma^{\prime} \cos \theta}{2} V_{\sigma \sigma^{\prime}} \operatorname{coth} \frac{V_{\sigma \sigma^{\prime}}}{2 T}, \\
S_{\dot{Q}_{F}}=\sum_{\sigma \sigma^{\prime}} \bar{G}_{\sigma \sigma^{\prime}} \frac{1+\sigma \sigma^{\prime} \cos \theta}{6}\left(\pi^{2} T^{2}+V_{\sigma \sigma^{\prime}}^{2}\right) V_{\sigma \sigma^{\prime}} \operatorname{coth} \frac{V_{\sigma \sigma^{\prime}}}{2 T},
\end{gathered}
$$
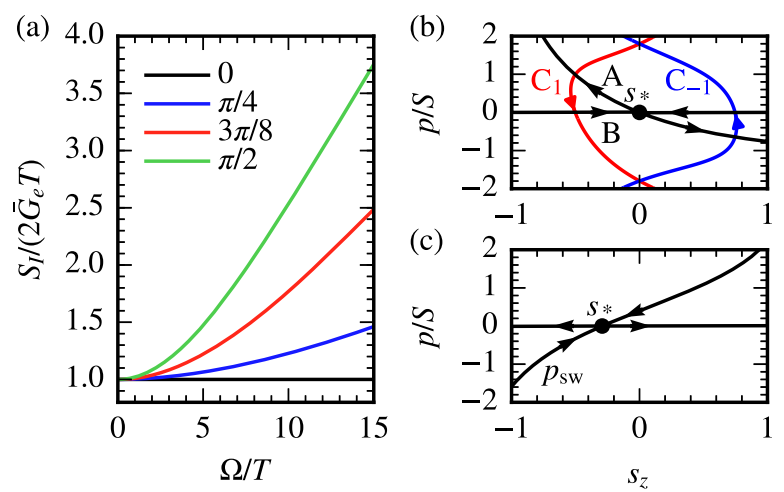

FIG. 2. (a) Noise in pumped charge current, for different tilt angles $\theta$ and precession speeds $\Omega$, for $P_{F^{\prime}}=P_{F z}=0.9$. (b) Semiclassical trajectories for $V=-\Omega_{\mathrm{ext}}, \quad P_{F^{\prime}}=1, \quad P_{F z}=1 / 2$, $\chi=\xi=0, T=0$. Shown are the $H=0$ lines $A B$ and the fixed point $s_{*}=\frac{1}{P_{F_{z}} P_{F^{\prime}}}+\frac{2 V}{P_{F^{\prime}} \Omega}$ (black). Measurement trajectories $C_{\tilde{\chi}}$ for $\tilde{\chi}=1$ (red) and $\tilde{\chi}=-1$ (blue) are also shown. (c) Trajectories with anharmonicity, $\Omega=\Omega_{\mathrm{an}} s_{z}, \quad V=1.5 \Omega_{\mathrm{an}}, \quad P_{F z}=0.1$, $P_{F^{\prime}}=1, T=0$.

where $V_{\sigma \sigma^{\prime}}=V+\Omega_{\sigma \sigma^{\prime}}$ and $T_{F}=T_{F^{\prime}}=T$. In the classical linear regime $V, \Omega<T$ the results reduce to a form dictated by the fluctuation-dissipation theorem and WiedemannFranz law, $S_{I}=2 \bar{G} T, \quad S_{\dot{Q}_{F}}=2 \bar{G} L_{0} T^{3}$, where $\bar{G}=$ $(d \bar{I} / d V)$ is the electrical dc conductance of the magnetic tunnel junction [38], and $L_{0}=\left(\pi^{2} / 3\right)\left(k_{B}^{2} / e^{2}\right)$ the Lorenz number. The presence of the angle-dependent frequencies is revealed in the quantum noise regime $\Omega>T$. The noise in the pumped current for $V=0$ is plotted in Fig. 2(a). The location of the quantum-classical crossover, typically at $\Omega \approx T$, is pushed up to higher precession frequencies as the tilt angle approaches $\theta=0$.

Spin torque induced fluctuating precession.-The above results are conditional on a specific value of $\theta$. For the full probability distribution, the distribution $P(\theta)$ would need to be known.

To find $P(\theta)=\int D\left[\theta^{c l}, \phi^{q}\right] e^{\left.i S\right|_{x=\xi=0}} \delta\left[\theta^{c l}(0)-\theta\right]$, we assume $\mathcal{S} \gg 1$ and take a semiclassical approximation. Defining $s_{z}=\cos \theta$ and $p=2 i \mathcal{S} \phi^{q}$, the action reads $\left.i S\right|_{\chi=\xi=0}=\int d t\left[p \dot{s}_{z}-H\left(p, s_{z}\right)\right]$, where $H=-i S_{T}$ is real for real $s_{z}, p$. The problem can then be analyzed as in Hamiltonian mechanics, $\dot{s}_{z}=\partial_{p} H, \dot{p}=-\partial_{s_{z}} H$ [34]. In a time-sliced discretization of the path integral, the $\delta$ restriction specifying the exact measured value adds a boundary condition $s_{z}(0)=s_{z 0}$ that removes one of the integration variables and saddle point equations. This allows for a discontinuity of $p$ at $t=0$; cf. Refs. [39,40]. The other boundary conditions are $p(t \rightarrow \pm \infty)=0$, so that relevant paths have integration constant $H=0$.

Consider now fluctuations close to an attractive fixed point $\tau\left(\theta_{*}\right)=0$ [cf. Fig. 1(b)]. For dynamics driven by an external field, it is located at $s_{z}=s_{*}=$ $\left(1 / P_{F^{\prime}} P_{F z}\right)+\left(2 V / P_{F^{\prime}} \Omega_{\text {ext }}\right)$, and it is attractive if $\tau^{\prime}\left(s_{*}\right)=$ $-\Omega P_{F z} P_{F^{\prime}}<0$. The phase space picture is shown in 

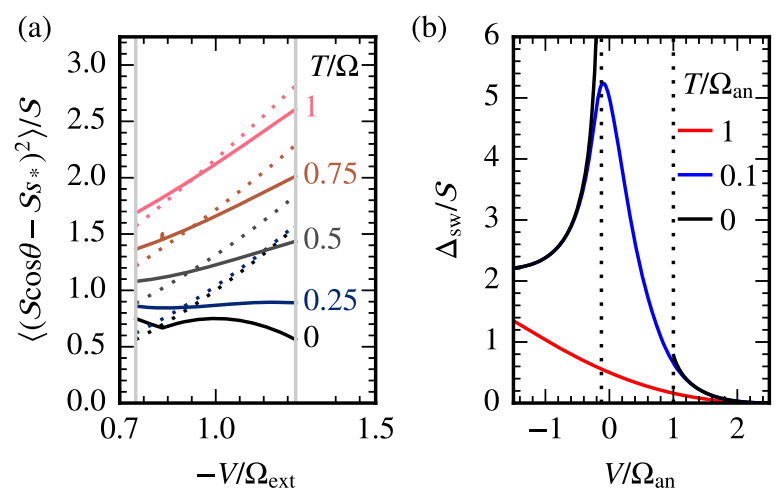

FIG. 3. (a) Normalized variance of the magnetization $z$ component in the steady state around the fixed point $s_{*}$, as a function of bias voltage and temperature for $P_{F^{\prime}}=1 / 2, P_{F z}=1 / 2$. Dotted lines indicate results where the energy shifts in the spin torque noise are neglected. (b) Switching exponent $\Delta_{\mathrm{sw}}$, for $P_{F^{\prime}}=1, P_{F z}=1 / 4$, and different temperatures and voltages. Dotted lines indicate the range $-\Omega_{\mathrm{an}} / 8<V<\Omega_{\mathrm{an}}$ where $\Delta_{s w}=\infty$ at $T=0$.

Fig. 2(b). Expanding around $p=0$ in terms of the torque $\tau$ and torque noise correlator $D$,

$$
\begin{gathered}
H \simeq\left[1-s_{z}^{2}\right]\left[\mathcal{S}^{-1} \tau\left(s_{z}\right) p-\mathcal{S}^{-2} D\left(s_{z}\right) p^{2}\right], \\
D\left(s_{z}\right)=\frac{1}{8} \sum_{\sigma \sigma^{\prime} \alpha= \pm} \frac{\left(1-\sigma \sigma^{\prime} s_{z}\right)^{2}}{1-s_{z}^{2}} \Gamma_{\sigma \sigma^{\prime} \alpha},
\end{gathered}
$$

where $\Gamma_{\sigma \sigma^{\prime} \alpha}=\int_{-\infty}^{\infty} \mathrm{d} \epsilon \Gamma_{\sigma \sigma^{\prime} \alpha}(\epsilon)=\bar{G}_{\sigma \sigma^{\prime}} \frac{1+\sigma \sigma^{\prime} s_{z}}{2} \alpha\left(V+\Omega_{\sigma \sigma^{\prime}}\right) /$ $\left[1-e^{-\alpha\left(V+\Omega_{\sigma \sigma^{\prime}}\right) / T}\right]$ for $T_{F}=T_{F^{\prime}}=T$. The fluctuation contribution comes from following path $A$ from $\left(s_{*}, 0\right)$ to $\left[s_{z 0}, \mathcal{S} \tau\left(s_{z 0}\right) / D\left(s_{z 0}\right)\right]$ :

$$
P(\cos \theta) \simeq N e^{\mathcal{S} \int_{s_{*}}^{\cos \theta} \mathrm{d} s_{z} \frac{\tau\left(s_{z}\right)}{D\left(s_{z}\right)}} \simeq N e^{\mathcal{S}^{I^{\prime}\left(s_{*}\right)}\left(\cos \theta-s_{*}\right)^{2}},
$$

where $N$ is a normalization constant. This agrees with Ref. [9] in the semiclassical limit $\mathcal{S} \gg 1$, except for the presence of the energy shifts $\propto \Omega_{\sigma \sigma^{\prime}}$ [11] in the spin torque noise correlator $D$, which are relevant in the quantum limit $\Omega \sim V \gg T$. The variance is plotted in Fig. 3(a).

Long measurement times.-For $t_{0} \gtrsim 1 / \theta$, the slow fluctuation of the magnetization contributes low-frequency noise to observables. This contribution is not small in $1 / \mathcal{S}$ : the typical excursion from the average position is small, $\delta s_{z} \propto \mathcal{S}^{-1 / 2}$, but it lasts for a long time $\tau_{m} \propto \mathcal{S}$, generating low-frequency noise $S_{I} \sim\left(\frac{d I}{d s_{z}} \delta s_{z}\right)^{2} \tau_{m}$. The situation is similar to noise induced in tunneling currents by temperature fluctuations on small islands [41].

We now find the result within the semiclassical approximation. The counting fields are switched on in the interval $0<t<t_{0}$, e.g., $\chi(t)=\theta(t) \theta\left(t_{0}-t\right) i \tilde{\chi}$. They make the semiclassical path to transition from branch $A$ to $B$ in the time interval $0<t<t_{0}$ following a trajectory $\mathrm{C}_{\tilde{\chi}, \tilde{\xi}}$ of constant $\left.H\right|_{\tilde{\chi}, \tilde{\xi}^{*}}$ Two such trajectories are shown in Fig. 2(a).

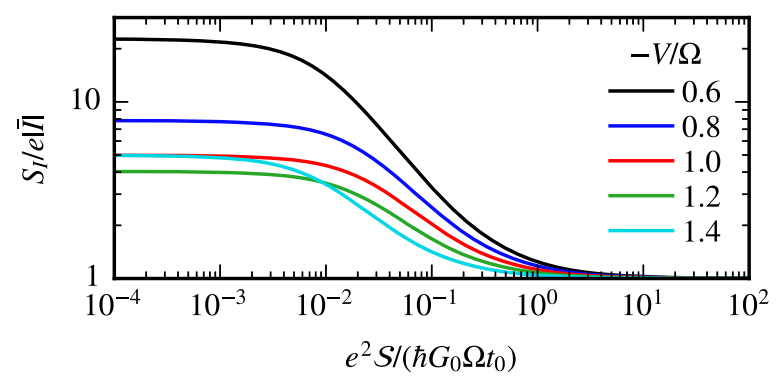

FIG. 4. Current noise $S_{I}$ as a function of the measurement bandwidth $1 / t_{0}$, for $P_{F^{\prime}}=1, P_{F z}=1 / 2, T=0$.

For simplicity, we consider the limit $T \ll|\Omega|,|V|$ with full polarization of the free magnet $P_{F^{\prime}}=1$. Then, close to $s_{*}$,

$H \simeq e^{\alpha \tilde{\chi}}\left[1-s_{*}^{2}\right]\left(\frac{\tau\left(s_{z}\right)}{\mathcal{S}} p-\frac{D\left(s_{*}\right)}{\mathcal{S}^{2}} p^{2}\right)-\Gamma\left(s_{z}\right)\left(e^{\alpha \tilde{\chi}}-1\right)$,

where $\alpha=\operatorname{sgn} V$ and $\Gamma\left(s_{z}\right)=\Gamma_{++\alpha}+\Gamma_{-+\alpha}$. For quadratic $H$, the Hamiltonian equations can be solved exactly (see Supplemental Material [28]). From this approach, we find the current noise,

$$
S_{I}=\Gamma\left(s_{*}\right)+4 \Gamma^{\prime}\left(s_{*}\right)^{2} \sigma_{s}^{2} \tau_{m}\left(1-\frac{1-e^{-t_{0} / \tau_{m}}}{t_{0} / \tau_{m}}\right),
$$

where $\tau_{m}=-\mathcal{S} /\left[\left(1-s_{*}^{2}\right) \tau^{\prime}\left(s_{*}\right)\right]$ is the slow time scale associated with the spin transfer torque and $\sigma_{s}^{2}=$ $-D\left(s_{*}\right) /\left[2 \mathcal{S} \tau^{\prime}\left(s_{*}\right)\right]$ the variance of the magnetization $z$ component in Eq. (14). The first term $\Gamma\left(s_{*}\right)$ in Eq. (16) is the Poissonian shot noise (10), and the second term originates from magnetization fluctuations. The dependence on the measurement time is shown in Fig. 4. The current noise at frequencies $\omega \sim \tau_{m}^{-1} \ll \Omega$ can be used to probe the dynamics and distribution of the magnetization.

Spin torque induced stochastic switching.-Magnetic anisotropy field $H_{\text {an }}$ results in an effective magnetic potential with two minima [see Fig. 1(c)], and the spin torque can induce switching between the two. Here, we take $H_{\text {ext }}=0$, and $\Omega=\gamma H_{\mathrm{an}} s_{z} \equiv \Omega_{\mathrm{an}} s_{z}$. The corresponding semiclassical Hamiltonian picture is shown in Fig. 2(c). An unstable fixed point $s_{*}=\left(1 / 2 P_{F^{\prime}} P_{F z}\right)$ $\left[1-\left(1+8 P_{F^{\prime}} P_{F z}^{2} V \Omega_{\mathrm{an}}^{-1}\right)^{1 / 2}\right]$ separates the two stable fixed points $s_{z}= \pm 1$. The leading exponent of the rate of switching from $s_{z}=-1$ to $s_{z}=1$ is [7],

$\Gamma_{\mathrm{sw}} \propto e^{-\Delta_{\mathrm{sw}}}=e^{\int_{-1}^{s_{*}} \mathrm{~d} s_{z} p_{\mathrm{sw}}\left(s_{z}\right)}, \quad H\left(s_{z}, p_{\mathrm{sw}}\left(s_{z}\right)\right)=0$,

where $p_{\text {sw }}\left(s_{z}\right)$ is shown in Fig. 2(c). The switching occurs deterministically $\left(\Delta_{\mathrm{sw}} \rightarrow 0\right)$ if $P_{F z} V>\frac{1+P_{F^{\prime}} P_{F z}}{2} \Omega_{\mathrm{an}}$ as $s_{z}=-1$ becomes unstable. At lower voltages, the switching is stochastic. Numerically computed results are shown in Fig. 3(b). At zero temperature, the switching is blocked [7] at $-\left(\Omega_{\mathrm{an}} / 8\right)<V<\Omega_{\mathrm{an}}$ for $P_{F^{\prime}}>P_{F z}$ and $-\Omega_{\mathrm{an}}<$ $V<\left(\Omega_{\text {an }} / 8\right)$ otherwise. This occurs because the transition 
rates $\Gamma_{\sigma \sigma^{\prime} \alpha}$ vanish for $\alpha\left(V+\Omega_{\sigma \sigma^{\prime}}\right) \leq 0$, and because the backaction $\propto \Omega_{\sigma \sigma^{\prime}}$ vanishes for $\sigma=-\sigma^{\prime}, s_{z} \rightarrow-1$ [42]. The latter constraint is due to the additional angle dependence in the spin torque, which traces back to the geometric phases [11] in the spin dynamics.

Discussion.-In conclusion, we have derived a Keldysh action (3), describing the stochastic charge and energy currents affected by a precessing magnetization. We obtain a fluctuation relation for the transferred charge, energy, and magnetization. The noise in the current at low temperatures displays features related to geometric phases, and its low frequency component reflects the magnetization fluctuations. Information about the spin torque noise is also contained in the switching probability of anisotropic magnets.

Our predictions are readily accessible in experiments probing spin pumping at low temperatures $T<\hbar \Omega / k_{B}$. Precession frequencies in the $10 \mathrm{GHz}$ range have been achieved [15,16], which translates to $T \lesssim 1 \mathrm{~K}$. The approach can be extended in a circuit theory fashion [38] to more complex microstructures than a single $F / F^{\prime}$ junction. Moreover, it allows for studying overheating effects, which are often relevant in spintronic devices. The temperature of the electrons in the nanomagnet can be considered to be set by a heat balance equation, and its stochastic variations [40,41] result in additional contributions to current noise, similar to those from the fluctuations of the magnetization.

We thank B. Nikolic and S. van Dijken for discussions. This work was supported by the MIUR-FIRB2013Project Coca (Grant No. RBFR1379UX), the Academy of Finland Centre of Excellence program (Project No. 284594) and the European Research Council (Grant No. 240362-Heattronics).

Note added.-Recently, a work [43] appeared considering a similar approach as here under different assumptions of the hierarchy of relaxation time scales.

[1] J. Slonczewski, J. Magn. Magn. Mater. 159, L1 (1996).

[2] Y. Tserkovnyak, A. Brataas, G. E. W. Bauer, and B. I. Halperin, Rev. Mod. Phys. 77, 1375 (2005).

[3] D. Ralph and M. Stiles, J. Magn. Magn. Mater. 320, 1190 (2008).

[4] W. F. Brown, Phys. Rev. 130, 1677 (1963).

[5] J. Foros, A. Brataas, Y. Tserkovnyak, and G. E. W. Bauer, Phys. Rev. Lett. 95, 016601 (2005).

[6] L. S. Levitov and G. B. Lesovik, JETP Lett. 58, 230 (1993).

[7] Y. Utsumi and T. Taniguchi, Phys. Rev. Lett. 114, 186601 (2015).

[8] D. M. Basko and M. G. Vavilov, Phys. Rev. B 79, 064418 (2009).

[9] A. L. Chudnovskiy, J. Swiebodzinski, and A. Kamenev, Phys. Rev. Lett. 101, 066601 (2008).

[10] G.-M. Tang and J. Wang, Phys. Rev. B 90, 195422 (2014).
[11] A. Shnirman, Y. Gefen, A. Saha, I. S. Burmistrov, M. N. Kiselev, and A. Altland, Phys. Rev. Lett. 114, 176806 (2015).

[12] J. Fransson and J.-X. Zhu, New J. Phys. 10, 013017 (2008).

[13] J.-X. Zhu, Z. Nussinov, A. Shnirman, and A. V. Balatsky, Phys. Rev. Lett. 92, 107001 (2004).

[14] L. Berger, Phys. Rev. B 54, 9353 (1996).

[15] S. I. Kiselev, J. C. Sankey, I. N. Krivorotov, N. C. Emley, R. J. Schoelkopf, R. A. Buhrman, and D. C. Ralph, Nature (London) 425, 380 (2003).

[16] W. H. Rippard, M. R. Pufall, S. Kaka, S. E. Russek, and T. J. Silva, Phys. Rev. Lett. 92, 027201 (2004).

[17] Y. Tserkovnyak, A. Brataas, and G. E. W. Bauer, Phys. Rev. Lett. 88, 117601 (2002).

[18] R. H. Koch, J. A. Katine, and J. Z. Sun, Phys. Rev. Lett. 92, 088302 (2004).

[19] S. Yakata, H. Kubota, T. Sugano, T. Seki, K. Yakushiji, A. Fukushima, S. Yuasa, and K. Ando, Appl. Phys. Lett. 95, 242504 (2009).

[20] G. E. Crooks, Phys. Rev. E 60, 2721 (1999).

[21] G. E. Crooks, Phys. Rev. E 61, 2361 (2000).

[22] U. Seifert, Rep. Prog. Phys. 75, 126001 (2012).

[23] M. Esposito, U. Harbola, and S. Mukamel, Rev. Mod. Phys. 81, 1665 (2009).

[24] J. Tobiska and Y. V. Nazarov, Phys. Rev. B 72, 235328 (2005).

[25] Y. Utsumi and H. Imamura, J. Phys. Conf. Ser. 200, 052030 (2010).

[26] R. López, J. S. Lim, and D. Sánchez, Phys. Rev. Lett. 108, 246603 (2012).

[27] C. Wang and D. E. Feldman, Phys. Rev. B 92, 064406 (2015).

[28] See Supplemental Material at http://link.aps.org/ supplemental/10.1103/PhysRevLett.118.237701, which includes Refs. [29-32], describing the derivation of Eq. (3), details of the semiclassical equations, and derivation of the fluctuation relations.

[29] A. G. Abanov and A. Abanov, Phys. Rev. B 65, 184407 (2002).

[30] M. Kindermann and S. Pilgram, Phys. Rev. B 69, 155334 (2004).

[31] S. Pilgram, K. E. Nagaev, and M. Büttiker, Phys. Rev. B 70, 045304 (2004).

[32] D. Andrieux and P. Gaspard, J. Chem. Phys. 121, 6167 (2004).

[33] M. Campisi, P. Hänggi, and P. Talkner, Rev. Mod. Phys. 83, 771 (2011).

[34] A. Kamenev and A. Levchenko, Adv. Phys. 58, 197 (2009).

[35] Y. Tserkovnyak, T. Moriyama, and J. Q. Xiao, Phys. Rev. B 78, 020401 (2008).

[36] A. Brataas, Y. Tserkovnyak, G. E. Bauer, and P. J. Kelly, Spin Current (Oxford University Press, New York, 2011), Chap. 8.

[37] For simplicity, we assume here that spin torque dominates magnetization damping. The presence of extra damping would lead to an additional term in $\alpha(\theta)$.

[38] D. Huertas-Hernando, Y. V. Nazarov, and W. Belzig, Phys. Rev. Lett. 88, 047003 (2002). 
[39] S. Pilgram, A. N. Jordan, E. V. Sukhorukov, and M. Büttiker, Phys. Rev. Lett. 90, 206801 (2003).

[40] T. T. Heikkilä and Y. V. Nazarov, Phys. Rev. Lett. 102, 130605 (2009).

[41] M. A. Laakso, T. T. Heikkilä, and Y. V. Nazarov, Phys. Rev. Lett. 104, 196805 (2010).
[42] The blocking is due to non-Gaussianity of the spin torque. The result neglects the exact quantization of spin and ignores, e.g., quantum tunneling.

[43] T. Ludwig, I. S. Burmistrov, Y. Gefen, and A. Shnirman, Phys. Rev. B 95, 075425 (2017). 